BI-TP 2006/07, hep-ph/0603048

\title{
Quark mass thresholds in QCD thermodynamics
}

\author{
Mikko Laint* and York Schrödel \\ Faculty of Physics, University of Bielefeld, D-33501 Bielefeld, Germany
}

\begin{abstract}
We discuss radiative corrections to how quark mass thresholds are crossed, as a function of the temperature, in basic thermodynamic observables such as the pressure, the energy and entropy densities, and the heat capacity of high temperature QCD. The indication from leading order that the charm quark plays a visible role at surprisingly low temperatures, is confirmed. We also sketch a way to obtain phenomenological estimates relevant for generic expansion rate computations at temperatures between the QCD and electroweak scales, pointing out where improvements over the current knowledge are particularly welcome.
\end{abstract}

PACS numbers: 11.10.Wx, 11.15.Bt, 12.38.Bx, 98.80.Cq

\section{INTRODUCTION}

Besides being of fundamental theoretical interest to finite temperature field theory, the thermodynamic pressure of the Standard Model, as a function of the temperature $T$ and of various chemical potentials $\mu_{i}$, has several potential phenomenological applications. Most notably it dictates, through the Einstein equations, the expansion rate of the radiation dominated Early Universe. The expansion rate in turn determines when various dark matter candidates decouple, thus fixing their relic densities: fine details of the pressure could become observable for instance if dark matter is made of electroweak scale WIMPs [1, 2] or of keV-scale sterile neutrinos [3, 4]. Furthermore, the pressure is in principle visible in the present-day spectrum of the gravitational wave background that was generated during the inflationary epoch [5]. More generally, the pressure incorporates the fact that the Standard Model possesses a trace anomaly, i.e. $T_{\mu}^{\mu} \neq 0$, which in turn can influence many kinds of gravity-related cosmological scenarios (for recent examples, see Refs. [6]).

Apart from cosmology, the pressure is potentially also relevant for the hydrodynamic expansion that the dense matter generated in current and upcoming heavy ion collision experiments may undergo. In this case there is some room for caution, however, since the issue of whether local thermodynamic equilibrium is reached remains controversial [7].

Given that the biggest theoretical challenges are related to strongly interacting particles, considerable efforts have been devoted to the determination of the QCD part of the pressure over a course of years. Denoting by $g$ the renormalised strong coupling constant, perturbative corrections to the non-interacting Stefan-Boltzmann law have been determined at relative orders $\mathcal{O}\left(g^{2}\right)[\underline{8}], \mathcal{O}\left(g^{3}\right)$ [9], $\mathcal{O}\left(g^{4} \ln (1 / g)\right)$ [10], $\mathcal{O}\left(g^{4}\right)$ [1], $\mathcal{O}\left(g^{5}\right)$ [12], and $\mathcal{O}\left(g^{6} \ln (1 / g)\right)$ [13], as a function of the number of colours, $N_{\mathrm{c}}$, and the number of massless quark flavours, $N_{\mathrm{f}}$. The first presently unknown order, $\mathcal{O}\left(g^{6}\right)$, contains non-perturbative coefficients [14, 15], but those can also be attacked [16, 17]. All orders of $g$ are available in the formal limit of large $N_{\mathrm{f}}[18$. These results have been extended to the case of finite quark chemical potentials [19, 20, 21] , and a similar computation has recently also been finalised for the weakly interacting part of the Standard Model, at temperatures higher than the electroweak scale 22|. Moreover, the fact that several orders are available allows to experiment with various kinds of resummations 23, 24].

Surprisingly, however, relatively little seems to be known about the dependence of the QCD pressure on the quark masses $m_{i}, i=1, \ldots, N_{\mathrm{f}}$. While the non-interacting Stefan-Boltzmann law is readily extended to this situation, it in fact appears that even the first non-trivial term, $\mathcal{O}\left(g^{2}\right)$, has not been exhaustively investigated in the literature (see, however, Ref. 25]). In principle this term has of course been available since almost 30 years [9], but in explicit form only in a renormalization scheme for quark masses which differs from the current standard, the $\overline{\mathrm{MS}}$ scheme. Furthermore, no general numerical evaluation of the basic integrals appearing has been presented, as far as we know. For $T=0$ but $\mu_{i} \neq 0$, the full $\mathcal{O}\left(g^{2}\right)$ analysis has also only been carried out recently [26].

Several probable reasons for the apparent lack of interest can surely be envisaged. First of all, the dependence on $N_{\mathrm{f}}$ is known to a high order in the massless case, and interpolating between integer values of $N_{\mathrm{f}}$ should give much of the information that we may need for the massive case. Second, including quark masses turns out to be technically cumbersome [9]. Third, there are several indications, for instance from considerations of the baryon chemical potential [19, 20] and of mesonic correlation lengths [27], that the convergence is much better in the quark sector than in

*Electronic address: laine@physik.uni-bielefeld.de

${ }^{\dagger}$ Electronic address: yorks@physik.uni-bielefeld.de 
the gluonic sector, so that the lowest non-trivial order may already provide sufficient accuracy. Nevertheless, we feel that the last assumption deserves to be checked, at least at the next-to-leading order $(\mathrm{NLO}) \mathcal{O}\left(g^{2}\right)$, and this is the purpose of the present paper.

In short, our general philosophy will then be to account for the gluonic contributions to the highest order available, $\mathcal{O}\left(g^{6} \ln (1 / g)\right)$, and consider the change that quarks with finite physical masses inflict on this result at $\mathrm{NLO}, \mathcal{O}\left(g^{2}\right)$. We do find that the quark mass effects at NLO are not too different from those at the leading order, $\mathcal{O}\left(g^{0}\right)$, such that the philosophy of terminating at $\mathcal{O}\left(g^{2}\right)$ is at least self-consistent. Nevertheless, we also outline the procedure for determining the quark mass dependence up to the order $\mathcal{O}\left(g^{6}\right)$.

Apart from the theoretical goals mentioned, we also wish to sketch certain phenomenological results in this paper. Consider the temperature evolution of an expanding system in the case of cosmology, for instance. Einstein equations then lead to (see, e.g., Ref. [28])

$$
\frac{1}{T} \frac{\mathrm{d} T}{\mathrm{~d} t}=-\sqrt{\frac{24 \pi e(T)}{m_{\mathrm{Pl}}^{2}}} \frac{s(T)}{c(T)},
$$

where $t$ is the time; we assumed the Universe to be flat $(k=0)$; and we ignored the cosmological constant. All the quantities appearing here follow from the pressure: $s(T)=p^{\prime}(T)$ is the entropy density, $e(T)=T s(T)-p(T)$ is the energy density, and $c(T)=e^{\prime}(T)=T p^{\prime \prime}(T)$ is the heat capacity. We wish to present our favoured "fits" for all these functions for temperatures between the QCD and electroweak scales, indicating where further work is required.

The plan of this paper is the following. We start by elaborating on the basic formalism in Sec. III discuss quark mass thresholds in Sec. III present a phenomenological evaluation of the various thermodynamic functions relevant for physical QCD in Sec. [V] include weakly interacting particles in Sec. V] and conclude in Sec. VI]

\section{BASIC FORMALISM}

In order to determine the basic thermodynamic quantities of the Standard Model, all of which can be derived from minus the grand canonical free energy density, or the pressure $p(T, \boldsymbol{\mu})$, where $\boldsymbol{\mu}$ collects together the various chemical potentials associated with conserved global charges, ${ }^{1}$ we make use of the framework of dimensionally reduced effective field theories [29, 30, 31]. This framework allows to organise the computation in a transparent way, and implements various resummations of higher order effects. We start by briefly reviewing certain aspects of the general framework for QCD; further details can be found in Ref. [13].

Dimensional reduction proceeds by first integrating out the "hard modes", with momenta or Matsubara frequencies of order $2 \pi T$. This produces an effective theory [29], called EQCD [31], which is a three-dimensional SU $\left(N_{\mathrm{c}}\right)$ gauge theory with a scalar field in the adjoint representation. The effective theory has a certain number of couplings, parametrised by functions denoted by $\alpha_{\mathrm{E} 1} \ldots \alpha_{\mathrm{E} 7}$ [contributing up to $\mathcal{O}\left(g^{6} \ln (1 / g)\right)$ ] and $\beta_{\mathrm{E} 1} \ldots \beta_{\mathrm{E} 5}[$ contributing at $\mathcal{O}\left(g^{6}\right)$ ] in Ref. [13]; in the following we explicitly specify the definitions for only a subset of them. These parameters contain all the information concerning the hard modes. Assuming the use of dimensional regularization, we denote by $\alpha_{\mathrm{Ei}}^{\overline{\mathrm{MS}}}, \beta_{\mathrm{Ei}}^{\overline{\mathrm{MS}}}$ parameters from which the $1 / \epsilon$-divergences have been removed by the $\overline{\mathrm{MS}}$-prescription.

To proceed, we need to specify explicitly the effective mass parameter $m_{3}^{2}$ and the effective gauge coupling $g_{3}^{2}$ of EQCD at NLO:

$$
\begin{aligned}
\hat{m}_{3}^{2} & \equiv \frac{m_{3}^{2}}{T^{2}} \equiv g^{2} \alpha_{\mathrm{E} 4}^{\overline{\mathrm{MS}}}+\frac{g^{4}}{(4 \pi)^{2}} \alpha_{\mathrm{E} 6}^{\overline{\mathrm{MS}}} \\
\hat{g}_{3}^{2} & \equiv \frac{g_{3}^{2}}{T} \equiv g^{2}+\frac{g^{4}}{(4 \pi)^{2}} \alpha_{\mathrm{E} 7}^{\overline{\mathrm{MS}}}
\end{aligned}
$$

Both parameters are renormalization group invariant up to the order computed, i.e., the dependence on the scale parameter $\bar{\mu}$ is of order $\mathcal{O}\left(g^{6}\right)$.

With this notation, the physical pressure of hot QCD can be written in the form

$$
p_{\mathrm{QCD}} \equiv p_{\text {hard }}+p_{\text {soft }}
$$

\footnotetext{
1 The notation $p(T, \boldsymbol{\mu})$ always implicitly refers to the ultraviolet finite difference $p(T, \boldsymbol{\mu})-p(0, \mathbf{0})$.
} 
where $p_{\text {hard }}$ represents the contribution of the hard modes (by definition containing both all direct hard contributions to the pressure, and all finite terms emerging from products like $\epsilon \cdot 1 / \epsilon$ ), while $p_{\text {soft }}$ represents the contribution of the soft modes. Up to the accuracy $\mathcal{O}\left(g^{6}\right), p_{\text {hard }}$ can conveniently be expressed as

$$
\begin{aligned}
\frac{p_{\text {hard }}}{T^{4}} & =\alpha_{\mathrm{E} 1}^{\overline{\mathrm{MS}}}+\hat{g}_{3}^{2} \alpha_{\mathrm{E} 2}^{\overline{\mathrm{MS}}}+\frac{\hat{g}_{3}^{4}}{(4 \pi)^{2}}\left(\alpha_{\mathrm{E} 3}^{\overline{\mathrm{MS}}}-\alpha_{\mathrm{E} 2}^{\overline{\mathrm{MS}}} \alpha_{\mathrm{E} 7}^{\overline{\mathrm{MS}}}-\frac{1}{4} d_{A} C_{A} \alpha_{\mathrm{E} 5}^{\overline{\mathrm{MS}}}\right)+ \\
& +\frac{\hat{g}_{3}^{6}}{(4 \pi)^{4}}\left\{\left[d_{A} C_{A}\left(\alpha_{\mathrm{E} 6}^{\overline{\mathrm{MS}}}-\alpha_{\mathrm{E} 4}^{\overline{\mathrm{MS}}} \alpha_{\mathrm{E} 7}^{\overline{\mathrm{MS}}}\right)-d_{A} C_{A}^{3}\left(\frac{43}{3}-\frac{27}{32} \pi^{2}\right)\right] \ln \frac{\bar{\mu}}{4 \pi T}+\Delta_{\text {hard }}\right\},
\end{aligned}
$$

where $d_{A} \equiv N_{\mathrm{c}}^{2}-1, C_{A} \equiv N_{\mathrm{c}}$, and we have separated a term on the last line which cancels the $\bar{\mu}$-dependence of $p_{\text {soft }}$ at $\mathcal{O}\left(g^{6}\right)$. The function $\Delta_{\text {hard }}$,

$$
\begin{aligned}
\Delta_{\mathrm{hard}} \equiv & {\left[d_{A} C_{A}\left(\alpha_{\mathrm{E} 6}^{\overline{\mathrm{MS}}}-\alpha_{\mathrm{E} 4}^{\overline{\mathrm{MS}}} \alpha_{\mathrm{E} 7}^{\overline{\mathrm{MS}}}\right)-d_{A} C_{A}^{3}\left(\frac{43}{3}-\frac{27}{32} \pi^{2}\right)\right] \ln \frac{4 \pi T}{\bar{\mu}}+} \\
& +\beta_{\mathrm{E} 1}^{\overline{\mathrm{MS}}}+2 \alpha_{\mathrm{E} 2}^{\overline{\mathrm{MS}}}\left(\alpha_{\mathrm{E} 7}^{\overline{\mathrm{MS}}}\right)^{2}-2 \alpha_{\mathrm{E} 3}^{\overline{\mathrm{MS}}} \alpha_{\mathrm{E} 7}^{\overline{\mathrm{MS}}}-\frac{1}{4} d_{A} C_{A}\left(\beta_{\mathrm{E} 2}^{\overline{\mathrm{MS}}}-\alpha_{\mathrm{E} 5}^{\overline{\mathrm{MS}}} \alpha_{\mathrm{E} 7}^{\overline{\mathrm{MS}}}+\alpha_{\mathrm{E} 4}^{\overline{\mathrm{MS}}} \beta_{\mathrm{E} 3}^{\overline{\mathrm{MS}}}\right),
\end{aligned}
$$

depends on $N_{\mathrm{c}}, N_{\mathrm{f}}, m_{i}, \mu_{i}$, and $\bar{\mu} / T$. The contributions of the soft modes are [13]

$$
\begin{aligned}
\frac{p_{\text {soft }}}{T^{4}} & =\frac{\hat{m}_{3}^{3}}{12 \pi} d_{A}-\frac{\hat{g}_{3}^{2} \hat{m}_{3}^{2}}{(4 \pi)^{2}} d_{A} C_{A}\left(\ln \frac{\bar{\mu}}{2 m_{3}}+\frac{3}{4}\right)-\frac{\hat{g}_{3}^{4} \hat{m}_{3}}{(4 \pi)^{3}} d_{A} C_{A}^{2}\left(\frac{89}{24}+\frac{\pi^{2}}{6}-\frac{11}{6} \ln 2\right)+ \\
& +\frac{\hat{g}_{3}^{6}}{(4 \pi)^{4}} d_{A} C_{A}^{3}\left[\left(\frac{43}{4}-\frac{491}{768} \pi^{2}\right) \ln \frac{\bar{\mu}}{2 m_{3}}+\left(\frac{43}{12}-\frac{157}{768} \pi^{2}\right) \ln \frac{\bar{\mu}}{2 C_{A} g_{3}^{2}}+\Delta_{\text {soft }}\right] .
\end{aligned}
$$

The function $\Delta_{\text {soft }}$ reads

$$
\Delta_{\mathrm{soft}}=\beta_{\mathrm{M}}+\beta_{\mathrm{G}}-\alpha_{\mathrm{E} 4}^{\overline{\mathrm{MS}}}\left[\frac{d_{A}+2}{4 C_{A}^{3}} \beta_{\mathrm{E} 4}^{\overline{\mathrm{MS}}}+\frac{2 d_{A}-1}{4 C_{A}^{4}} \beta_{\mathrm{E} 5}^{\overline{\mathrm{MS}}}\right],
$$

where $\beta_{\mathrm{M}}$ can be found in Ref. [32], and a numerical estimate of $\beta_{\mathrm{G}}$ in Ref. [17].

Let us stress that the formulae presented apply independently of whether quark masses are included or not: all quark mass effects can be incorporated in the perturbative functions $\alpha_{\mathrm{E} 1}^{\overline{\mathrm{MS}}} \ldots \alpha_{\mathrm{E} 7}^{\overline{\mathrm{MS}}}, \beta_{\mathrm{E} 1}^{\overline{\mathrm{MS}}} \ldots \beta_{\mathrm{E} 5}^{\overline{\mathrm{MS}}}$. In particular, the nonperturbative numerical value $\beta_{\mathrm{G}}$ and the contribution from the Debye scale $\beta_{\mathrm{M}}$ in Eq. (8) are "universal".

In the following, we refer to the various orders of the weak-coupling expansion according to the power of $\hat{g}_{3}, \hat{m}_{3}$ that appear, with the rule $\mathcal{O}\left(\hat{m}_{3}\right)=\mathcal{O}\left(\hat{g}_{3}\right)=\mathcal{O}(g)$. In other words, " $\mathcal{O}\left(g^{n}\right)$ " denotes $\mathcal{O}\left(\hat{g}_{3}^{n-k} \hat{m}_{3}^{k}\right)$ in the expression constituted by Eqs. (41), (5), (7). If $\hat{g}_{3}^{2}, \hat{m}_{3}^{2}$ were to be re-expanded in terms of $g^{2}$, the result of Ref. [13] would be reproduced up to $\mathcal{O}\left(g^{6}\right)$. In practice, however, it is advisable to keep the result in an unexpanded form, because this makes it more manageable, and because the unexpanded form introduces resummations of higher order contributions.

\section{QUARK MASS THRESHOLDS IN THE PRESSURE}

In the absence of an explicit $\mathcal{O}\left(g^{6} \ln (1 / g)\right)$-computation with $m_{i} \neq 0$, we are forced to estimate the effects of finite quark masses by other means. In order to do this, we take as the starting point the limit $N_{\mathrm{f}}=0$, where all quarks are treated as infinitely heavy. Subsequently, the masses of a number $N_{\mathrm{f}}$ of them are lowered to their physical values. In this process the pressure at any given temperature increases. The goal is then to estimate the "correction factors" that describe this increase. Note that this philosophy corresponds to the "unquenching" of quark effects that is regularly attempted in lattice simulations.

The philosophy just suggested is of course not unique. For instance, one could also start from the "opposite" limit, taking as the starting point the pressure for a fixed $N_{\mathrm{f}}$, but with vanishing quark masses, and then increase the masses. In fact, one could perhaps even define an effective non-integer massless $N_{\mathrm{f}}$ by evaluating the massive $\alpha_{\mathrm{E} 1}^{\overline{\mathrm{MS}}}$ (Eq. (11) below), fitting it to the massless formula (Eq. (A.1) in Ref. [13]), and using the resulting $N_{\mathrm{f}}$ in the massless result of $\mathcal{O}\left(g^{6} \ln (1 / g)\right)$ [33]. We prefer, however, to take here the infinitely massive case $N_{\mathrm{f}}=0$ as the reference point, since this limit can be treated with more confidence than the chiral limit, as we describe in the next section, and since this limit offers us a convenient way to probe the convergence of our recipe, as we now explain.

The simplest thinkable way to estimate the correction factors due to non-infinite quark masses would be to multiply the $N_{\mathrm{f}}=0$ result with the change indicated by the Stefan-Boltzmann law, i.e. by $\alpha_{\mathrm{E} 1}^{\overline{\mathrm{MS}}}\left(N_{\mathrm{f}}\right) / \alpha_{\mathrm{E} 1}^{\overline{\mathrm{MS}}}(0)$ [2]. The first nontrivial improvement of this philosophy is then to determine the functions $\alpha_{\mathrm{E} 1}^{\overline{\mathrm{MS}}}, \alpha_{\mathrm{E} 2}^{\overline{\mathrm{MS}}}, \alpha_{\mathrm{E} 7}^{\overline{\mathrm{MS}}}$ in the general massive case, 

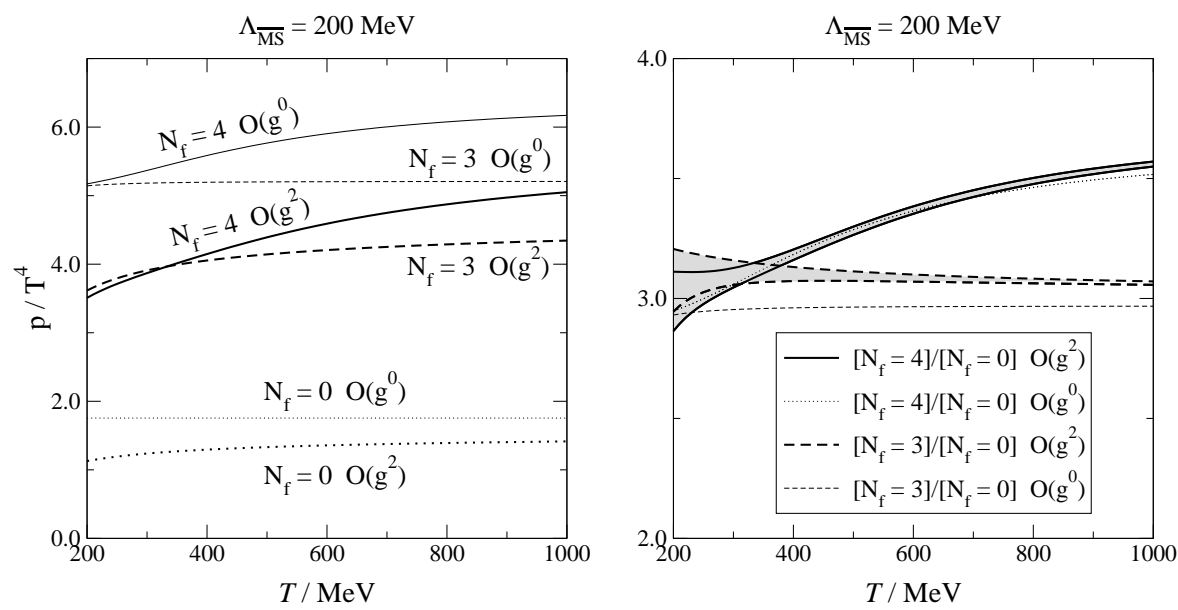

FIG. 1: Left: the pressure for $N_{\mathrm{f}}=0,3,4$, at $\mathcal{O}\left(g^{0}\right)$ and $\mathcal{O}\left(g^{2}\right)$. Right: the "correction factors" accounting for the effects of quarks, at $\mathcal{O}\left(g^{0}\right)$ and $\mathcal{O}\left(g^{2}\right)$ (cf. Eq. (10) ). They grey bands indicate the effect of $\overline{\mathrm{MS}}$ scheme scale variations by a factor $0.5 \ldots 2.0$ around the "optimal" value. It is observed that while the $\mathcal{O}\left(g^{2}\right)$ corrections are of order $20 \ldots 30 \%$ in the pressure, they are of order $5 \%$ in the correction factors for $N_{\mathrm{f}}=3$, and even less for the physical case $N_{\mathrm{f}}=4$.

which allows us to evaluate the order $\mathcal{O}\left(g^{2}\right)$ result for the pressure, viz.

$$
\frac{p_{\mathrm{QCD}}}{T^{4}} \approx \alpha_{\mathrm{E} 1}^{\overline{\mathrm{MS}}}+\hat{g}_{3}^{2} \alpha_{\mathrm{E} 2}^{\overline{\mathrm{MS}}} .
$$

Afterwards, we may modify the $N_{\mathrm{f}}=0$ result with a correction factor,

$$
\frac{\left[\alpha_{\mathrm{E} 1}^{\overline{\mathrm{MS}}}+\hat{g}_{3}^{2} \alpha_{\mathrm{E} 2}^{\overline{\mathrm{MS}}}\right]\left(N_{\mathrm{f}}\right)}{\left[\alpha_{\mathrm{E} 1}^{\overline{\mathrm{MS}}}+\hat{g}_{3}^{2} \alpha_{\mathrm{ES} 2}^{\overline{\mathrm{MS}}}\right](0)} .
$$

Comparing the outcome of this $\mathcal{O}\left(g^{2}\right)$ recipe with the corresponding $\mathcal{O}\left(g^{0}\right)$ recipe allows to probe the convergence. Note that it is important to also determine $\alpha_{\mathrm{E} 7}^{\overline{\mathrm{MS}}}$, since only this way can the renormalisation scale that appears in $\hat{g}_{3}^{2}$ be reasonably fixed (cf. Eq. (3)).

We thus proceed to compute $\alpha_{\mathrm{E} 1}^{\overline{\mathrm{MS}}}, \alpha_{\mathrm{E} 2}^{\overline{\mathrm{MS}}}, \alpha_{\mathrm{E} 7}^{\overline{\mathrm{MS}}}$. We do this in full generality, keeping $N_{\mathrm{f}}, N_{\mathrm{c}}$, the quark masses $m_{i}$, and the chemical potentials $\mu_{i}$ as free parameters. The quark masses and the strong gauge coupling are renormalised in the $\overline{\mathrm{MS}}$ scheme. Some details concerning the computation are collected in Appendix A. As final results, we obtain

$$
\begin{aligned}
\alpha_{\mathrm{E} 1}^{\overline{\mathrm{MS}}}= & d_{A} \frac{\pi^{2}}{45}+4 C_{A} \sum_{i=1}^{N_{\mathrm{f}}} F_{1}\left(\frac{m_{i}^{2}}{T^{2}}, \frac{\mu_{i}}{T}\right), \\
\alpha_{\mathrm{E} 2}^{\overline{\mathrm{MS}}}= & -\frac{d_{A} C_{A}}{144}-d_{A} \sum_{i=1}^{N_{\mathrm{f}}}\left\{\frac{1}{6} F_{2}\left(\frac{m_{i}^{2}}{T^{2}}, \frac{\mu_{i}}{T}\right)\left[1+6 F_{2}\left(\frac{m_{i}^{2}}{T^{2}}, \frac{\mu_{i}}{T}\right)\right]+\right. \\
& \left.+\frac{m_{i}^{2}}{4 \pi^{2} T^{2}}\left(3 \ln \frac{\bar{\mu}}{m_{i}}+2\right) F_{2}\left(\frac{m_{i}^{2}}{T^{2}}, \frac{\mu_{i}}{T}\right)-\frac{2 m_{i}^{2}}{T^{2}} F_{4}\left(\frac{m_{i}^{2}}{T^{2}}, \frac{\mu_{i}}{T}\right)\right\}, \\
\alpha_{\mathrm{E} 7}^{\overline{\mathrm{MS}}}= & \frac{22 C_{A}}{3}\left[\ln \left(\frac{\bar{\mu} e^{\gamma_{E}}}{4 \pi T}\right)+\frac{1}{22}\right]-\frac{2}{3} \sum_{i=1}^{N_{\mathrm{f}}}\left[2 \ln \frac{\bar{\mu}}{m_{i}}+F_{3}\left(\frac{m_{i}^{2}}{T^{2}}, \frac{\mu_{i}}{T}\right)\right],
\end{aligned}
$$

where the functions $F_{1}, \ldots, F_{4}$ and some of their properties are detailed in Appendix B.

To estimate the numerical importance of the $\mathcal{O}\left(g^{2}\right)$ corrections, we need to assign a value to all the parameters that appear. Following a simple-minded logic, we use 1-loop running,

$$
g^{2}(\bar{\mu})=\frac{24 \pi^{2}}{\left(11 C_{A}-4 T_{F}\right) \ln \left(\bar{\mu} / \Lambda_{\overline{\mathrm{MS}}}\right)}, \quad m_{i}(\bar{\mu})=m_{i}\left(\bar{\mu}_{\mathrm{ref}}\right)\left[\frac{\ln \left(\bar{\mu}_{\mathrm{ref}} / \Lambda_{\overline{\mathrm{MS}}}\right)}{\ln \left(\bar{\mu} / \Lambda_{\overline{\mathrm{MS}}}\right)}\right]^{\frac{9 C_{F}}{11 C_{A}-4 T_{F}}},
$$

where $T_{F}=N_{\mathrm{f}} / 2, C_{F}=\left(N_{\mathrm{c}}^{2}-1\right) / 2 N_{\mathrm{c}}, \bar{\mu}_{\text {ref }} \equiv 2 \mathrm{GeV}$. The quark masses at $\bar{\mu}=\bar{\mu}_{\text {ref }}$ are taken from Ref. 34]. To choose $\bar{\mu}$, we apply the principle of minimal sensitivity criterion for the parameter $\hat{g}_{3}^{2}$, as suggested in Ref. [35]. Furthermore, for illustration, we set $\Lambda_{\overline{\mathrm{MS}}} \equiv 200 \mathrm{MeV}$. 
The outcome of this procedure is shown in Fig. 1 for $\mu_{i}=0$. It is observed that while the $\mathcal{O}\left(g^{2}\right)$ corrections are of order $20 \ldots 30 \%$ in the pressure (left panel), the "correction factors", i.e. the ratios in Eq. (10), only contain $\mathcal{O}\left(g^{2}\right)$ corrections of order $5 \%$ for $N_{\mathrm{f}}=3$, and even less for the physical case $N_{\mathrm{f}}=4$ (right panel). This implies that the quark mass dependence of the pressure probably converges faster than the weak-coupling expansion as a whole.

Finally, we note from Fig. 1(right) that the charm quark contribution starts to be visible already at fairly low temperatures. At leading order, the quark mass dependence is determined by the function $F_{1}$ (cf. Eq. (11)), which at low temperatures has the familiar classical form

$$
F_{1}\left(\frac{m^{2}}{T^{2}}, 0\right) \approx\left(\frac{m}{2 \pi T}\right)^{\frac{3}{2}} \exp \left(-\frac{m}{T}\right) .
$$

It is observed that $F_{1}$ obtains $5 \%$ of its asymptotic value $7 \pi^{2} / 720$ at temperatures as low as $T \approx m / 5$. (For the precise numerical values of $F_{1}$, see Fig. 5. As Fig. प(right) shows, the onset of a visible charm mass dependence is postponed to about $T \sim 350 \mathrm{MeV}$ at $\mathcal{O}\left(g^{2}\right)$, but the basic pattern remains unchanged.

\section{PHENOMENOLOGICAL RESULTS FOR QCD}

We now move from fairly well-defined expressions towards phenomenology. The goal is to present, where possible, an educated numerical guess for the physical QCD pressure. We set all chemical potentials to zero in the following.

The general philosophy we adopt is that, for temperatures above the deconfinement transition, the weak-coupling expansion needs to be evaluated up to the order where the dominant contributions from all the different scales $\left(2 \pi T, g T, g^{2} T\right)$ have made their entrances. There is some support for such a recipe from a number of non-trivial observables [36, 37]. In practice, this means that the QCD pressure would need to be evaluated up to $\mathcal{O}\left(g^{6}\right)$.

Unfortunately, one of the $\mathcal{O}\left(g^{6}\right)$ terms, parametrised by $\beta_{\mathrm{E} 1}^{\overline{\mathrm{MS}}}$ in Sec. III remains completely unknown for the moment even in the massless limit: it is a function of $N_{\mathrm{f}}$ and requires a perturbative 4-loop computation. This introduces a certain unknown "constant" into the prediction. We propose to fix the constant by the following recipe.

Let us start by considering the case $N_{\mathrm{f}}=0, N_{\mathrm{c}}=3$. Then the expressions in Sec. on $\bar{\mu} / T$ (through $\left.\alpha_{\mathrm{E} 1}^{\overline{\mathrm{MS}}} \ldots \alpha_{\mathrm{E} 7}^{\overline{\mathrm{MS}}}, \beta_{\mathrm{E} 1}^{\overline{\mathrm{MS}}} \ldots \beta_{\mathrm{E} 5}^{\overline{\mathrm{MS}}}\right)$, and on $\bar{\mu} / \Lambda_{\overline{\mathrm{MS}}}\left(\right.$ through $\left.g^{2}(\bar{\mu})\right)$. It so happens that the dependence on $\bar{\mu}$, which formally cancels up to the order of the computation, is numerically non-monotonous (see, e.g., Ref. [23]), so that the specific choice is not terribly important, as long as we are close to the extremum. In practice we choose $\bar{\mu} / T$ according to the principle of minimal sensitivity criterion for the parameter $\hat{g}_{3}^{2}$, as already mentioned. Thereby the results only depend on $T / \Lambda_{\overline{\mathrm{MS}}}$ and on the unknown $\mathcal{O}\left(g^{6}\right)$ terms, contained in $\Delta_{\text {hard }}$ and $\Delta_{\text {soft }}$, defined in Eqs. (5), (7). It is important to note that once $\bar{\mu} / T$ has been fixed, the $\Delta$ 's can be treated as temperature-independent constants. It is furthermore convenient to combine them into a single term, ${ }^{2}$

$$
\Delta_{\text {hard }}+d_{A} C_{A}^{3} \Delta_{\text {soft }} \equiv d_{A} C_{A}^{3} \Delta .
$$

In order to now eliminate the dependence on $\Delta$, we "match" the perturbative prediction to $4 \mathrm{~d}$ lattice simulation results for the case $N_{\mathrm{f}}=0$, where the continuum limit has been reached with reasonable precision [38, 39]. It should be stressed that this step is purely phenomenological: in principle $\Delta$ is computable from the theory. On the other hand, there is every reason to expect that results obtained through the dimensionally reduced framework do match $4 \mathrm{~d}$ lattice results as soon as $T \gtrsim 2 T_{\mathrm{c}}$, where $T_{\mathrm{c}}$ is the temperature of the deconfinement phase transition (see, e.g., Refs. [36], [40]-[42]). Moreover, that a family of functions specified by a single parameter should match a given function for a whole range of argument values, provides for a non-trivial consistency check.

Now, lattice results are usually presented in terms of $T / T_{\mathrm{c}}$, rather than $T / \Lambda_{\overline{\mathrm{MS}}}$. We thus need a value for $T_{\mathrm{c}} / \Lambda_{\overline{\mathrm{MS}}}$; we use $T_{\mathrm{c}} / \Lambda_{\overline{\mathrm{MS}}} \approx 1.20$ which appears to be consistent with all independent determinations (cf. Ref. [37], Sec. 4.2). After this choice, an excellent match can be obtained (we do this by minimising the difference squared of the function values in the range $T>3 T_{\mathrm{c}}$ ), with a value $\Delta \approx-3.287$ (cf. Fig. [2). In the following we will take the cubic spline interpolation shown in Fig. [2 as the "starting point", which will then be "corrected" by the effects of quarks.

To now include quarks, we simply multiply the result just obtained by the correction factor in Eq. (10). We should expect this construction to work the better the higher the temperature, but surely at least $T>200 \mathrm{MeV}$ is required.

It needs to be noted, however, that like in Fig. 1 the evaluation of the correction factor necessitates fixing $\Lambda_{\overline{\mathrm{MS}}}$ in physical units. This exercise is non-trivial. We again choose a purely phenomenological but rather convenient

\footnotetext{
${ }^{2}$ We note that $\Delta$ differs by a certain constant from a similar constant employed in the figures of Ref. [13].
} 


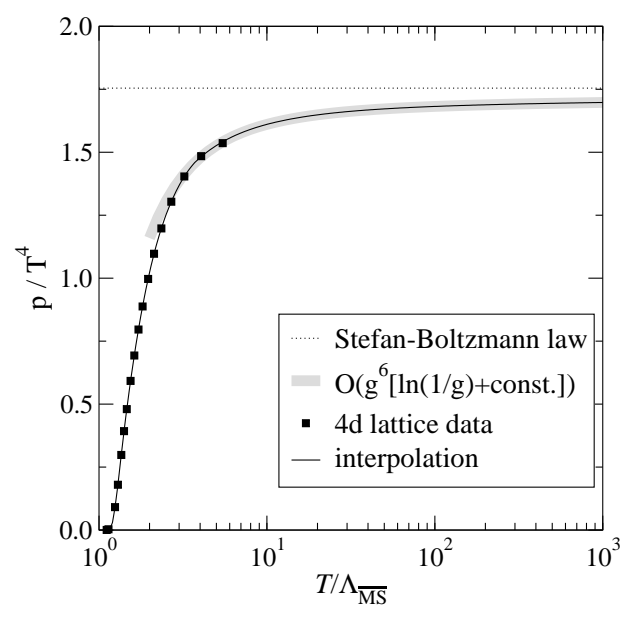

FIG. 2: A phenomenological interpolating curve (solid line) for the QCD pressure at $N_{\mathrm{f}}=0$. In the perturbative curve (grey band) the unknown $\mathcal{O}\left(g^{6}\right)$ constant has been adjusted so that lattice data (closed squares $[38]$ ) is matched, once $T>3.6 \Lambda_{\overline{\mathrm{MS}}}$.

procedure, which makes use of the pressure produced by the full set of hadronic resonances [34]. Indeed, it has been demonstrated recently that if the resonance masses are tuned to correspond to the quark masses accessible to current lattice simulations, the resulting "resonance gas pressure" works surprisingly well even for temperatures deep into the crossover region [43].

Thus, we tune $\Lambda_{\overline{\mathrm{MS}}}$ such that our analytic recipe and the first derivative thereof match the resonance gas result (in which the temperature is automatically measured in physical units) at a certain temperature. Examples are shown in Fig. 31 (left). The values of $\Lambda_{\overline{\mathrm{MS}}}$ that result depend slightly on $N_{\mathrm{f}}$ and on variations of quark masses within their experimental errors, but the typical range is $\Lambda_{\overline{\mathrm{MS}}} \approx 175 \ldots 180 \mathrm{MeV}$. We should stress that this matching is of course rather arbitrary, but it does produce qualitatively reasonable results with, for instance, an inflection point on the side $T>T_{\mathrm{c}} \sim 175 \mathrm{MeV}$, as suggested by lattice results [44]-[47].

Naturally, the resonance gas results cannot really be trusted in quantitative detail for temperatures above, say, $150 \mathrm{MeV}$. Therefore, for a certain interval (which we choose to be $T=150 \ldots 350 \mathrm{MeV}$, and shade in all figures), the results remain to be established by lattice simulations. The matter becomes even more urgent, when one considers derivatives of the pressure, to which we now turn.

Apart from the pressure, its first and second derivatives play an important role, as already mentioned in connection with Eq. (11). There are various ways of presenting the information contained in these derivatives: we may for instance parametrise the physical observables $e(T), s(T), c(T)$ through effective numbers of bosonic degrees of freedom,

$$
g_{\mathrm{eff}}(T) \equiv \frac{e(T)}{\left[\frac{\pi^{2} T^{4}}{30}\right]}, \quad h_{\mathrm{eff}}(T) \equiv \frac{s(T)}{\left[\frac{2 \pi^{2} T^{3}}{45}\right]}, \quad i_{\mathrm{eff}}(T) \equiv \frac{c(T)}{\left[\frac{2 \pi^{2} T^{3}}{15}\right]},
$$

in terms of which Eq. (11) becomes

$$
\frac{3}{2} \sqrt{\frac{5}{\pi^{3}}} \frac{m_{\mathrm{Pl}}}{T^{3}} \frac{\mathrm{d} T}{\mathrm{~d} t}=-\frac{\sqrt{g_{\mathrm{eff}}(T)} h_{\mathrm{eff}}(T)}{i_{\mathrm{eff}}(T)},
$$

or we can consider dimensionless ratios like

$$
\begin{aligned}
w(T) & \equiv \frac{p(T)}{e(T)}=\frac{p(T)}{T p^{\prime}(T)-p(T)}, \\
c_{s}^{2}(T) & \equiv \frac{p^{\prime}(T)}{e^{\prime}(T)}=\frac{p^{\prime}(T)}{T p^{\prime \prime}(T)}=\frac{s(T)}{c(T)} .
\end{aligned}
$$

Both the "equation-of-state" $w(T)$ and the sound speed squared $c_{s}^{2}(T)$ equal $1 / 3$ in the non-interacting limit. The deviation of the parameter $w(T)$ from $1 / 3$ is proportional to the trace anomaly, sometimes also called the interaction measure.

Results for all of these quantities, based on our interpolation, are shown in Fig. 3(middle, right). It can be seen that quantities involving derivatives show a significant amount of structure around the QCD crossover, even if there 

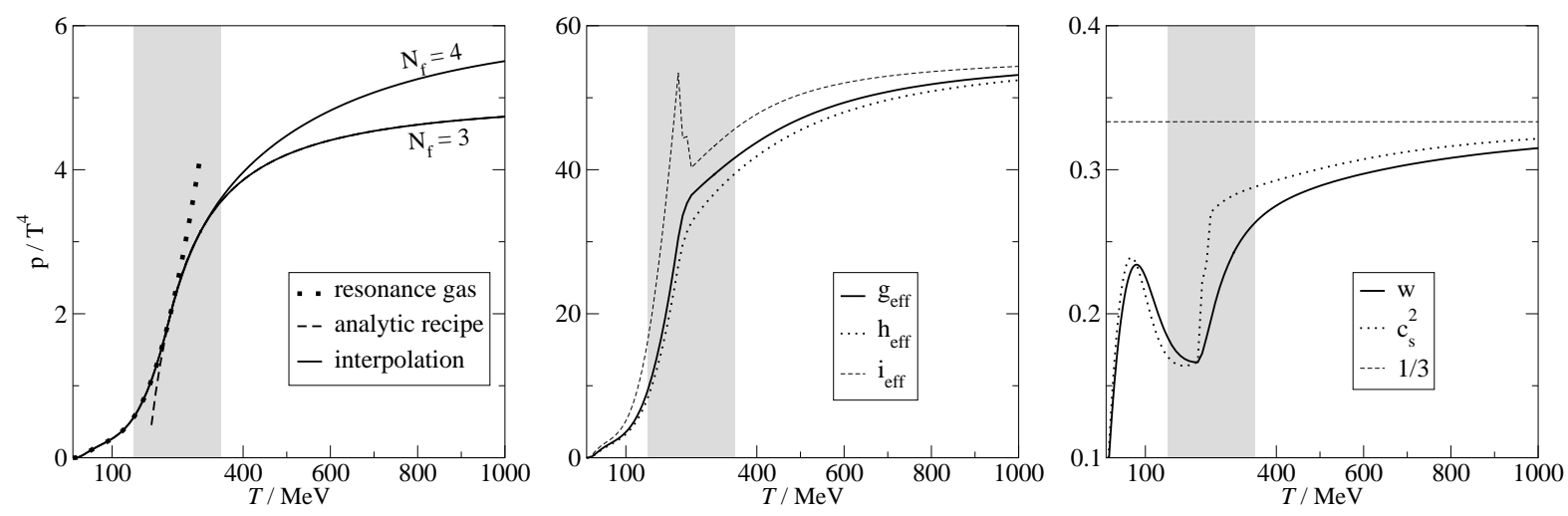

FIG. 3: Left: Phenomenological interpolating curves (solid lines) for the QCD pressure at $N_{\mathrm{f}}=3,4$. The shaded interval corresponds to the transition region where the results can be reliably determined with lattice simulations only. Middle: $g_{\mathrm{eff}}, h_{\mathrm{eff}}, i_{\mathrm{eff}}$ as defined in Eq. (17), for $N_{\mathrm{f}}=4$. Right: the equation-of-state parameter $w$ and the speed of sound squared $c_{s}^{2}$, for the same system.

were no singularities. We remark that to smooth the behaviour we have evaluated $p(T, \boldsymbol{\mu})$ with a relatively sparse temperature grid in the critical region.

Clearly, it is important to correct the results in the "shaded region" by using results from future lattice simulations of the type in Refs. 44]- 47]. In particular, the recent Refs. [46, 47] display direct results for $c_{s}^{2}$ and $w$, respectively. Unfortunately, it does not appear that these results would be useful for our present purposes: they for instance fail to reproduce the significant rise in $w$ and $c_{s}^{2}$ that is seen in Fig. B(right) at temperatures down from the critical one, displaying rather a much deeper dip (down to $\sim 0.1$ ) around the critical region, and then rising at most slightly as the temperature is lowered. Therefore, it could be feared that the dip itself is affected by the unphysically heavy quark masses that are used in the simulations.

We finally comment on the peak visible in $i_{\text {eff }}$ in Fig. 3 (middle). While the details are of course not captured by our phenomenological recipe, the fact that a peak exists in the heat capacity is not unexpected for rapid crossovers; in second order phase transition, the heat capacity even diverges as $T \rightarrow T_{\mathrm{c}}$.

\section{PHENOMENOLOGICAL RESULTS FOR THE STANDARD MODEL}

While in heavy ion collisions at most strongly interacting particles have time to thermalise, the expansion rate is much smaller in cosmology, so that all Standard Model degrees of freedom do reach thermal equilibrium, and remain thermalised until neutrino decoupling at around $T \sim \mathrm{MeV}$. Therefore, their contributions need to be added to the QCD pressure. In practice, we count gluons and the four lightest quarks as the QCD degrees of freedom, while the bottom and top quark are treated as part of the "weakly interacting" sector, so that the result splits into a sum of two terms.

We will assume that it is sufficient to treat the weakly interacting sector at 1-loop level. That is, we construct the free energy density $f$ in the presence of a Higgs expectation value $v$, temperature $T$, and chemical potentials $\mu_{i}$, according to

$$
f(v, T, \boldsymbol{\mu})=-\frac{1}{2} \nu^{2}(\bar{\mu}) v^{2}+\frac{1}{4} \lambda(\bar{\mu}) v^{4}+\sum_{i} \sigma_{i} \mathcal{J}_{i}\left(m_{i}(v), T, \mu_{i}\right)
$$

where the sum extends over all physical degrees of freedom, with their proper degeneracies; $\sigma_{i}=+1(-1)$ for bosons (fermions); and the tree-level masses $m_{i}(v)$ depend on $v$ in the standard way (it is sufficient at this order to work in unitary gauge). For scalar $\left(\mathcal{J}_{\mathrm{s}}\right)$, vectors $\left(\mathcal{J}_{\mathrm{v}}\right)$ and fermions $\left(\mathcal{J}_{\mathrm{f}}\right)$,

$$
\begin{aligned}
& \mathcal{J}_{\mathrm{s}}=-\frac{m^{4}}{64 \pi^{2}}\left(\ln \frac{\bar{\mu}^{2}}{m^{2}}+\frac{3}{2}\right)+\frac{T^{4}}{4 \pi^{2}} \int_{0}^{\infty} \mathrm{d} x x^{\frac{1}{2}} \ln \left(1-e^{-\sqrt{x+y}}\right)_{y=\frac{m^{2}}{T^{2}}} \\
& \mathcal{J}_{\mathrm{v}}=-\frac{m^{4}}{64 \pi^{2}}\left(\ln \frac{\bar{\mu}^{2}}{m^{2}}+\frac{5}{6}\right)+\frac{T^{4}}{4 \pi^{2}} \int_{0}^{\infty} \mathrm{d} x x^{\frac{1}{2}} \ln \left(1-e^{-\sqrt{x+y}}\right)_{y=\frac{m^{2}}{T^{2}}} \\
& \mathcal{J}_{\mathrm{f}}=-\frac{m^{4}}{64 \pi^{2}}\left(\ln \frac{\bar{\mu}^{2}}{m^{2}}+\frac{3}{2}\right)+T^{4} F_{1}\left(\frac{m^{2}}{T^{2}}, \frac{\mu}{T}\right) .
\end{aligned}
$$



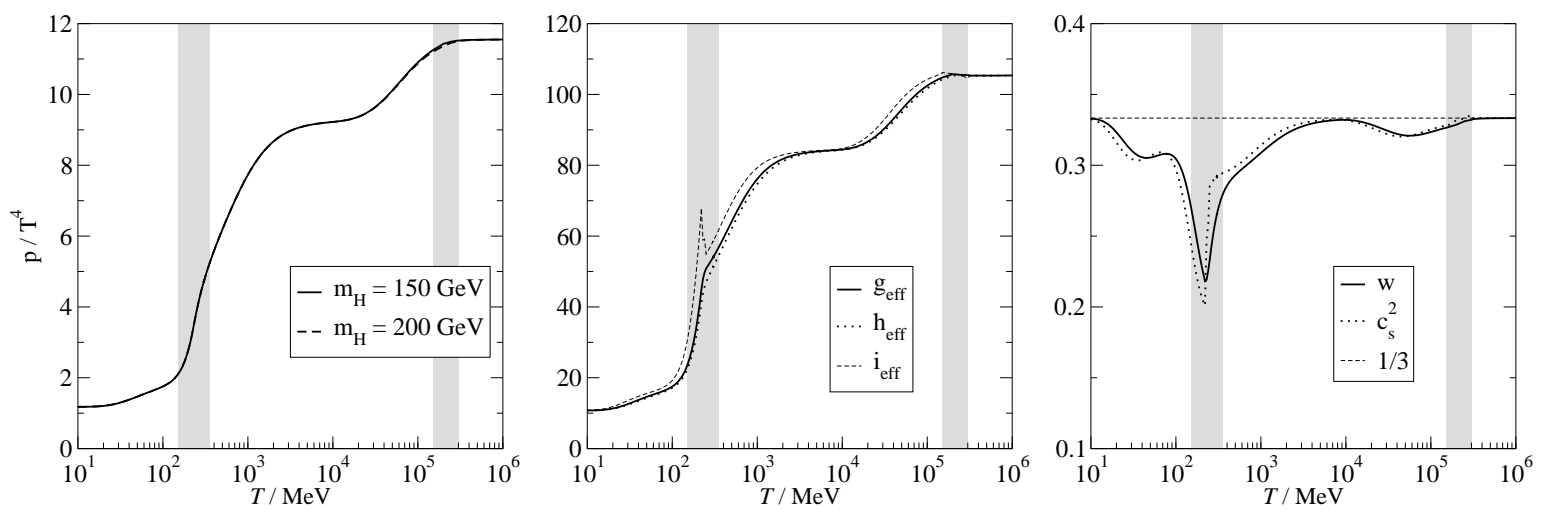

FIG. 4: Left: The Standard Model pressure for $m_{H}=150 \mathrm{GeV}, 200 \mathrm{GeV}$. The shaded intervals correspond to the QCD and electroweak transition regions. Middle: $g_{\text {eff }}, h_{\text {eff }}, i_{\text {eff }}$ as defined in Eq. (17), for $m_{H}=150$ GeV. Right: the equation-of-state parameter $w$ and the speed of sound squared $c_{s}^{2}$, for the same system. Various sources of uncertainties are discussed in the text.

The renormalised pressure is then given by

$$
p(T, \boldsymbol{\mu})=\min _{v} f(v, 0, \mathbf{0})-\min _{v} f(v, T, \boldsymbol{\mu}) .
$$

The renormalised pressure depends on a number of parameters defined in the $\overline{\mathrm{MS}}$ scheme: the Higgs potential parameters $\nu^{2}(\bar{\mu}), \lambda(\bar{\mu})$ (cf. Eq. (21)); and the weak gauge and the top and bottom Yukawa couplings $g_{w}^{2}(\bar{\mu}), h_{t}^{2}(\bar{\mu})$, $h_{b}^{2}(\bar{\mu})$ (through the tree-level masses). The first four of these we express through the Fermi constant and the $W^{ \pm}$, Higgs, and top pole masses, employing the explicit 1-loop relations listed in Ref. [30], while the last one is fixed through the bottom mass in the $\overline{\mathrm{MS}}$ scheme [34]. Given that the electroweak theory contains a multitude of scales, both zero temperature and thermal, we simply choose a fixed $\bar{\mu}=100 \mathrm{GeV}$ for the weakly interacting part of the pressure (we have varied the scale by a factor $0.5 \ldots 2.0$, and seen that the dependence is invisible on our resolution).

Let us remark that Eq. (25) suffers from the problem that it leads to a first order electroweak phase transition at a certain temperature, while there is none if the theory is treated more carefully [48, 49]. In practice this does not lead to any serious complications, however: we again smooth the behaviour by evaluating $p(T, \boldsymbol{\mu})$ with a sparse temperature grid around the critical region. In our figures, we shade the corresponding temperature interval, where our estimates are qualitative at best.

With these reservations, the whole Standard Model pressure, and the parameters defined in Eqs. (17), (19), (20), are shown in Fig. [4.

At temperatures above the electroweak scale, our results are already very close to the ideal gas results. Recently, higher loop corrections in this region have been considered in some detail 22]. The authors find a rather more significant deviation from the ideal gas value, due for instance to the top Yukawa coupling. We have not implemented these corrections, however, since they would require a correspondingly higher order computation in the broken symmetry phase. Though such a computation exists in principle up to 2-loop level [50], we did not consider the non-trivial challenges posed by its numerical evaluation for general masses to be worth tackling at present, given that in the quantities plotted in Fig. 4(right), the 2-loop contributions (which do not contribute to the trace anomaly on the symmetric phase side) are expected to largely cancel out. Nevertheless, it would be important to finalise this computation, if physics is made with the temperature interval $T=10 \ldots 100 \mathrm{GeV}$, where the $W^{ \pm}, Z^{0}$ bosons and top quark cross their mass thresholds.

Finally, once a definite Higgs model is available, it will of course be important to carry out lattice simulations for the transition region. Fortunately, for the electroweak theory this can be achieved within the dimensionally reduced effective theory [30], whereby also all fermions with their physical Yukawa couplings can be fully accounted for.

\section{CONCLUSIONS}

The functional dependence of the QCD pressure at a high temperature $T$ on most of the parameters of the theory (number of colours $N_{\mathrm{c}}$, number of active massless flavours $N_{\mathrm{f}}$, and quark chemical potentials $\mu_{i}$ ) is known up to relative order $\mathcal{O}\left(g^{6} \ln (1 / g)\right)$, while the dependence on the quark masses $m_{i}$ has gained much less interest. The purpose of this note has been to study whether it indeed is justified to consider the effects of finite non-zero quark masses at the level of the non-interacting Stefan-Boltzmann law (i.e. $\mathcal{O}\left(g^{0}\right)$ ), as has been the standard procedure. For this purpose, 
we have determined the corrections of order $\mathcal{O}\left(g^{2}\right)$ in full generality in the $\overline{\mathrm{MS}}$ scheme, and presented a numerical evaluation of all the integrals that appear in this result.

We find that while the $\mathcal{O}\left(g^{2}\right)$ corrections are in general $20 \ldots 30 \%$ (Fig. I(left)), they are numerically at most $5 \%$ for the quark mass dependence (Fig. 1(right)). This is perhaps in accord with previous observations according to which quarks are fairly perturbative as soon as they are deconfined, even though gluons do display strong interactions up to very high temperatures.

Finally, we have sketched educated "guesses" for the thermodynamic quantities that play a role in various physical contexts, for temperatures between the QCD and electroweak scales. For the case of heavy ion collisions, in particular, it is perhaps relevant to keep in mind that if the charm quark does thermalise, it has a rather significant effect even at relatively low temperatures (Fig. 3(left)). Of course, it is by no means clear whether such a thermalization should take place in practice [51].

In order to improve on our QCD results, the missing perturbative $\mathcal{O}\left(g^{6}\right)$ computations and, naturally, lattice simulations in the transition region, with physical values of the quark masses, remain to be completed.

For the full Standard Model, we have presented similar guesses for the various quantities that are relevant for expansion rate and particle decoupling computations (Figs. 44). Although the deviations from previous phenomenological estimates that have appeared in the literature [1,2] are in general fairly small, we nevertheless hope that our results help for their part to gauge the systematic uncertainties that still exist in these quantities.

In particular, we have stressed the need for repeating the computations of Ref. [22] in the broken symmetry phase and, of course, the need for effective theory lattice simulations in the transition region, once the electroweak model / Higgs mass is known.

\section{Acknowledgements}

We acknowledge useful discussions with P. Huovinen, K. Kajantie, K. Rummukainen, M. Shaposhnikov, and A. Vuorinen, and thank J. Engels for providing lattice data from Ref. [38].

\section{APPENDIX A: OUTLINE OF THE COMPUTATION}

In this Appendix we present a few details for the computation leading to Eqs. (11)- (13). We concentrate on the fermionic contributions; the bosonic ones are elementary.

We write the fermionic contributions in terms of the renormalised gauge coupling $g^{2}$ and the renormalised quark masses $m_{i}, i=1, \ldots, N_{\mathrm{f}}$. The master integrals emerging are

$$
\begin{aligned}
I_{\mathrm{b}} & \equiv \sum_{P_{\mathrm{b}}} \frac{1}{P_{\mathrm{b}}^{2}}, \\
J_{\mathrm{f}}(m, \mu) & \equiv \frac{1}{2} \sum_{P_{\mathrm{f}}} \ln \left(\tilde{P}_{\mathrm{f}}^{2}+m^{2}\right), \\
I_{\mathrm{f}}(m, \mu) & \equiv \sum_{P_{\mathrm{f}}} \frac{1}{\tilde{P}_{\mathrm{f}}^{2}+m^{2}}, \\
H_{\mathrm{f}}(m, \mu) & \equiv \sum_{P_{\mathrm{f}}, Q_{\mathrm{f}}} \frac{1}{\left(\tilde{P}_{\mathrm{f}}^{2}+m^{2}\right)\left(\tilde{Q}_{\mathrm{f}}^{2}+m^{2}\right)\left(\tilde{P}_{\mathrm{f}}-\tilde{Q}_{\mathrm{f}}\right)^{2}},
\end{aligned}
$$

where $P_{\mathrm{b}}, P_{\mathrm{f}}$ denote bosonic and fermionic Matsubara four-momenta, respectively, and $\tilde{P}_{\mathrm{f}} \equiv P_{\mathrm{f}}+(-i \mu, \mathbf{0})$ includes the chemical potential $\mu$. With this notation, the fermionic contributions to the parameters of interest are

$$
\begin{aligned}
T^{4} \alpha_{\mathrm{E} 1}^{\mathrm{f}} & =4 C_{A} \sum_{i=1}^{N_{\mathrm{f}}} J_{\mathrm{f}}\left(m_{i}, \mu_{i}\right) \\
T^{4} \alpha_{\mathrm{E} 2}^{\mathrm{f}} & =2 C_{A} \hat{\delta}_{1} m^{2} \sum_{i=1}^{N_{\mathrm{f}}} m_{i}^{2} I_{\mathrm{f}}\left(m_{i}, \mu_{i}\right)+ \\
& +d_{A} \sum_{i=1}^{N_{\mathrm{f}}}\left\{\frac{d-1}{2}\left[2 I_{\mathrm{b}}-I_{\mathrm{f}}\left(m_{i}, \mu_{i}\right)\right] I_{\mathrm{f}}\left(m_{i}, \mu_{i}\right)+2 m_{i}^{2} H_{\mathrm{f}}\left(m_{i}, \mu_{i}\right)\right\}
\end{aligned}
$$




$$
\frac{1}{(4 \pi)^{2}} \alpha_{\mathrm{E} 7}^{\mathrm{f}}=\hat{\delta}_{1} g^{2}+\frac{2}{3} \sum_{i=1}^{N_{\mathrm{f}}} \frac{\mathrm{d} I_{\mathrm{f}}\left(m_{i}, \mu_{i}\right)}{\mathrm{d} m_{i}^{2}},
$$

where $\hat{\delta}_{1} m^{2}, \hat{\delta}_{1} g^{2}$ are counterterms defined by writing the bare mass parameter and gauge coupling as $m_{B i}^{2}=m_{i}^{2}(1+$ $\left.g^{2} \hat{\delta}_{1} m^{2}\right), g_{B}^{2}=g^{2}\left(1+g^{2} \hat{\delta}_{1} g^{2}\right)$; it is understood that only the fermionic part of $\hat{\delta}_{1} g^{2}$ is considered; and $d \equiv 3-2 \epsilon$.

The next step is the evaluation of the Matsubara sums appearing in the master integrals. For the 1-loop structures (Eqs. A1- A3), it is straightforward to obtain

$$
\begin{aligned}
I_{\mathrm{b}} & =\int \frac{\mathrm{d}^{d} \mathbf{p}}{(2 \pi)^{d}} \frac{1}{2|\mathbf{p}|}\left[1+2 n_{\mathrm{B}}(|\mathbf{p}|)\right], \\
J_{\mathrm{f}}(m, \mu) & =\int \frac{\mathrm{d}^{d} \mathbf{p}}{(2 \pi)^{d}} \frac{-\mathbf{p}^{2}}{2 d E}\left[1-n_{\mathrm{F}}(E-\mu)-n_{\mathrm{F}}(E+\mu)\right]_{E=\sqrt{\mathbf{p}^{2}+m^{2}}}, \\
I_{\mathrm{f}}(m, \mu) & =\int \frac{\mathrm{d}^{d} \mathbf{p}}{(2 \pi)^{d}} \frac{1}{2 E}\left[1-n_{\mathrm{F}}(E-\mu)-n_{\mathrm{F}}(E+\mu)\right]_{E=\sqrt{\mathbf{p}^{2}+m^{2}}},
\end{aligned}
$$

where we have carried out a partial integration after the sum in $J_{\mathrm{f}}$, and

$$
n_{\mathrm{B}}(E) \equiv \frac{1}{e^{\beta E}-1}, \quad n_{\mathrm{F}}(E) \equiv \frac{1}{e^{\beta E}+1} .
$$

As is well known, the momentum integral in Eq. A8) can be carried out explicitly, $I_{\mathrm{b}}=\pi^{d / 2} T^{d} \Gamma(1-d / 2) \zeta(2-d) / 2 \pi^{2} T$, but the ones in Eqs. (A9), A10 with $m, \mu \neq 0$ cannot in general be integrated in closed form.

For the genuine 2-loop integral $H_{\mathrm{f}}$ in Eq. (A4) the sums are slightly more complicated, so we give here some details. The method we employ follows the standard procedure [9] (see also Refs. [52, 53]). The twofold sum over the Matsubara modes is first written as a threefold sum with a Kronecker delta-function, and the delta-function is then written as $\delta\left(p_{0}\right)=T \int_{0}^{\beta} \mathrm{d} x \exp \left(i p_{0} x\right)$. The sums can now be performed:

$$
\begin{aligned}
& T \sum_{p_{\mathrm{b}}} \frac{e^{i p_{\mathrm{b}} x}}{p_{\mathrm{b}}^{2}+E^{2}}=\frac{1}{2 E} n_{\mathrm{B}}(E)\left[e^{(\beta-x) E}+e^{x E}\right], \\
& T \sum_{p_{\mathrm{f}}} \frac{e^{i \tilde{p}_{\mathrm{f}} x}}{\tilde{p}_{\mathrm{f}}^{2}+E^{2}}=\frac{1}{2 E}\left[n_{\mathrm{F}}(E+\mu) e^{(\beta-x) E+\beta \mu}-n_{\mathrm{F}}(E-\mu) e^{x E}\right],
\end{aligned}
$$

where $p_{\mathrm{b}}, p_{\mathrm{f}}$ denote the bosonic and fermionic Matsubara frequencies, respectively; and $\tilde{p}_{\mathrm{f}}=p_{\mathrm{f}}-i \mu$. The integral over $x$ is then simple. All the exponents appearing can be written in terms of inverses of the distribution functions $n_{\mathrm{B}}$, $n_{\mathrm{F}}$, and multiplying with their explicit appearances, we are left with at most quadratic products of the distribution functions, and fractions containing the three "energies".

The fractions containing the energies can be organized in a transparent form, once we introduce the zero-temperature objects

$$
\begin{aligned}
H_{\mathrm{vac}}\left(m_{1}^{2}, m_{2}^{2}, m_{3}^{2}\right) & =\int \frac{\mathrm{d}^{4-2 \epsilon} P}{(2 \pi)^{4-2 \epsilon}} \int \frac{\mathrm{d}^{4-2 \epsilon} Q}{(2 \pi)^{4-2 \epsilon}} \frac{1}{\left[P^{2}+m_{1}^{2}\right]\left[Q^{2}+m_{2}^{2}\right]\left[(P-Q)^{2}+m_{3}^{2}\right]}, \\
\Pi\left(Q^{2} ; m_{1}^{2}, m_{2}^{2}\right) & =\int \frac{\mathrm{d}^{4-2 \epsilon} P}{(2 \pi)^{4-2 \epsilon}} \frac{1}{\left[P^{2}+m_{1}^{2}\right]\left[(P-Q)^{2}+m_{2}^{2}\right]}, \\
\Delta\left(Q^{2} ; m_{3}^{2}\right) & =\frac{1}{Q^{2}+m_{3}^{2}} .
\end{aligned}
$$

Indeed, carrying out the integrals over $P_{0}, Q_{0}$ in these functions, one obtains similar energy fractions. Making furthermore use of the $\mathrm{O}(4-2 \epsilon)$ rotational invariance of the $Q$-dependence in Eq. A15, which is present once also the integration over $\mathbf{p}$ is performed, the various fractions can be identified with each other.

In order to write the subsequent result in a compact but generic form, we introduce the notation

$$
E_{i} \equiv \sqrt{\mathbf{p}_{i}^{2}+m_{i}^{2}}, \quad P_{i} \equiv\left(E_{i}, \mathbf{p}_{i}\right), \quad P_{i} \cdot P_{j} \equiv E_{i} E_{j}-\mathbf{p}_{i} \cdot \mathbf{p}_{j},
$$

and denote

$$
n_{ \pm}\left(E_{i}\right) \equiv \begin{cases}n_{\mathrm{B}}\left(E_{i}\right) & \text { for bosons }\left(\equiv E_{3}\right) \\ -n_{\mathrm{F}}\left(E_{i} \pm \mu\right) & \text { for fermions }\left(\equiv E_{1}, E_{2}\right)\end{cases}
$$


Then, allowing for generality for three different masses, like in Eq. A14,

$$
\begin{aligned}
H_{\mathrm{f}} & =H_{\mathrm{vac}}\left(m_{1}^{2}, m_{2}^{2}, m_{3}^{2}\right)+ \\
& +\sum_{i \neq j \neq k} \sum_{\sigma= \pm 1} \int \frac{\mathrm{d}^{d} \mathbf{p}_{i}}{(2 \pi)^{d}} \frac{n_{\sigma}\left(E_{i}\right)}{2 E_{i}} \Pi\left(-m_{i}^{2} ; m_{j}^{2}, m_{k}^{2}\right)+ \\
& +\sum_{i \neq j \neq k} \sum_{\sigma, \tau= \pm 1} \int \frac{\mathrm{d}^{d} \mathbf{p}_{i}}{(2 \pi)^{d}} \int \frac{\mathrm{d}^{d} \mathbf{p}_{j}}{(2 \pi)^{d}} \frac{n_{\sigma}\left(E_{i}\right) n_{\tau}\left(E_{j}\right)}{4 E_{i} E_{j}} \Delta\left[-\left(\sigma P_{i}-\tau P_{j}\right)^{2} ; m_{k}^{2}\right],
\end{aligned}
$$

where $\sum_{i \neq j \neq k} \equiv \sum_{(i, j, k)=(1,2,3),(2,3,1),(3,1,2)}$. Individual terms in this sum may contain infrared poles (or, after performing some of the integrations in complex plane, imaginary parts), but the expression as a whole is finite and real for $\epsilon \neq 0$.

We return now to the case of physical interest $\left(m_{1}^{2}=m_{2}^{2} \equiv m^{2} ; m_{3}^{2} \equiv 0\right)$, and ignore all temperature-independent terms. We note, furthermore, that the contribution originating from the last term in Eq. (A19) for $(i, j, k)=(3,1,2)$, contains $\Delta\left[-\left(P_{3}+P_{1}\right)^{2} ; m^{2}\right]+\Delta\left[-\left(P_{3}-P_{1}\right)^{2} ; m^{2}\right]$ which vanishes, given that $P_{1}^{2}+P_{3}^{2}=m^{2}$. The same is true for $(i, j, k)=(2,3,1)$. This leaves us with

$$
\begin{aligned}
H_{\mathrm{f}}(m, \mu) & =[\text { temperature-independent terms }]+ \\
& +I_{\mathrm{b}} \Pi\left(0 ; m^{2}, m^{2}\right)+2 I_{\mathrm{f}}(m ; \mu) \Pi\left(-m^{2} ; m^{2}, 0\right)+ \\
& +\int \frac{\mathrm{d}^{d} \mathbf{p}_{1}}{(2 \pi)^{d}} \int \frac{\mathrm{d}^{d} \mathbf{p}_{2}}{(2 \pi)^{d}} \frac{n_{-}\left(E_{1}\right) n_{+}\left(E_{2}\right)+n_{+}\left(E_{1}\right) n_{-}\left(E_{2}\right)}{8 E_{1} E_{2}} \frac{1}{\mathbf{p}_{1} \cdot \mathbf{p}_{2}-m^{2}-E_{1} E_{2}}+ \\
& +\int \frac{\mathrm{d}^{d} \mathbf{p}_{1}}{(2 \pi)^{d}} \int \frac{\mathrm{d}^{d} \mathbf{p}_{2}}{(2 \pi)^{d}} \frac{n_{-}\left(E_{1}\right) n_{-}\left(E_{2}\right)+n_{+}\left(E_{1}\right) n_{+}\left(E_{2}\right)}{8 E_{1} E_{2}} \frac{1}{\mathbf{p}_{1} \cdot \mathbf{p}_{2}-m^{2}+E_{1} E_{2}}
\end{aligned}
$$

where we substituted $\mathbf{p}_{1} \rightarrow-\mathbf{p}_{1}$ in the last term. We can still perform the integration over $z \equiv \mathbf{p}_{1} \cdot \mathbf{p}_{2} /\left|\mathbf{p}_{1}\right|\left|\mathbf{p}_{2}\right|$, leaving a rapidly convergent integral over $\left|\mathbf{p}_{1}\right|,\left|\mathbf{p}_{2}\right|$.

The final step is the expansion in $\epsilon$. The only temperature-dependent ultraviolet divergences are in the factorised terms on the second row in Eq. (A20). Adding together with the contributions from the other master integrals, as specified in Eqs. (A5)-(A7), a straightforward computation reproduces the fermionic parts of Eqs. (11)-(13).

\section{APPENDIX B: FUNCTIONS DETERMINING THE MASS DEPENDENCE}

The functions that appear in Eqs. (11)-(13) are defined as

$$
\begin{aligned}
F_{1}(y, \hat{\mu}) \equiv & \frac{1}{24 \pi^{2}} \int_{0}^{\infty} \mathrm{d} x\left[\frac{x}{x+y}\right]^{\frac{1}{2}}\left[\hat{n}_{\mathrm{F}}(\sqrt{x+y}-\hat{\mu})+\hat{n}_{\mathrm{F}}(\sqrt{x+y}+\hat{\mu})\right] x, \\
F_{2}(y, \hat{\mu}) \equiv & \frac{1}{8 \pi^{2}} \int_{0}^{\infty} \mathrm{d} x\left[\frac{x}{x+y}\right]^{\frac{1}{2}}\left[\hat{n}_{\mathrm{F}}(\sqrt{x+y}-\hat{\mu})+\hat{n}_{\mathrm{F}}(\sqrt{x+y}+\hat{\mu})\right], \\
F_{3}(y, \hat{\mu}) \equiv & -\int_{0}^{\infty} \mathrm{d} x\left[\frac{x}{x+y}\right]^{\frac{1}{2}}\left[\hat{n}_{\mathrm{F}}(\sqrt{x+y}-\hat{\mu})+\hat{n}_{\mathrm{F}}(\sqrt{x+y}+\hat{\mu})\right] \frac{1}{x}, \\
F_{4}(y, \hat{\mu}) \equiv & \frac{1}{(4 \pi)^{4}} \int_{0}^{\infty} \mathrm{d} x_{1} \int_{0}^{\infty} \mathrm{d} x_{2} \frac{1}{\sqrt{x_{1}+y} \sqrt{x_{2}+y}} \times \\
& \times\left\{\left[\hat{n}_{\mathrm{F}}\left(\sqrt{x_{1}+y}-\hat{\mu}\right) \hat{n}_{\mathrm{F}}\left(\sqrt{x_{2}+y}+\hat{\mu}\right)+\hat{n}_{\mathrm{F}}\left(\sqrt{x_{1}+y}+\hat{\mu}\right) \hat{n}_{\mathrm{F}}\left(\sqrt{x_{2}+y}-\hat{\mu}\right)\right] \times\right. \\
& \times \ln \left[\frac{\sqrt{x_{1}+y} \sqrt{x_{2}+y}+y-\sqrt{x_{1} x_{2}}}{\sqrt{x_{1}+y} \sqrt{x_{2}+y}+y+\sqrt{x_{1} x_{2}}}\right]+ \\
& +\left[\hat{n}_{\mathrm{F}}\left(\sqrt{x_{1}+y}-\hat{\mu}\right) \hat{n}_{\mathrm{F}}\left(\sqrt{x_{2}+y}-\hat{\mu}\right)+\hat{n}_{\mathrm{F}}\left(\sqrt{x_{1}+y}+\hat{\mu}\right) \hat{n}_{\mathrm{F}}\left(\sqrt{x_{2}+y}+\hat{\mu}\right)\right] \times \\
& \left.\times \ln \left[\frac{\sqrt{x_{1}+y} \sqrt{x_{2}+y}-y+\sqrt{x_{1} x_{2}}}{\sqrt{x_{1}+y} \sqrt{x_{2}+y}-y-\sqrt{x_{1} x_{2}}}\right]\right\}
\end{aligned}
$$


where

$$
\hat{n}_{\mathrm{F}}(x) \equiv \frac{1}{e^{x}+1} .
$$

These functions are related to the functions $J_{\mathrm{f}}, I_{\mathrm{f}}$ and $H_{\mathrm{f}}$ defined in Eqs. (A2)-A4): the medium-modified part of $J_{\mathrm{f}}$ reads $T^{4} F_{1}$ for $\epsilon=0$; the medium-modified part of $I_{\mathrm{f}}$ reads $-T^{2} F_{2}$ for $\epsilon=0$; the medium-modified part of $\mathrm{d} I_{\mathrm{f}} / \mathrm{d} m^{2}$ reads $-F_{3} /(4 \pi)^{2}$ for $\epsilon=0$; and the "non-factorizable" part of $H_{\mathrm{f}}$ (the last two terms in Eq. A20) reads $T^{2} F_{4}$ for $\epsilon=0$. The functions $F_{1}, F_{2}, F_{3}$ are related by

$$
F_{2}(y, \hat{\mu})=-2 \frac{\partial F_{1}(y, \hat{\mu})}{\partial y}, \quad F_{3}(y, \hat{\mu})=(4 \pi)^{2} \frac{\partial F_{2}(y, \hat{\mu})}{\partial y} .
$$

The functions introduced possess some solvable limiting values. For $y \rightarrow 0$,

$$
\begin{aligned}
& F_{1}(0, \hat{\mu})=\frac{7 \pi^{2}}{720}+\frac{\hat{\mu}^{2}}{24}+\frac{\hat{\mu}^{4}}{48 \pi^{2}}, \\
& F_{2}(0, \hat{\mu})=\frac{1}{24}+\frac{\hat{\mu}^{2}}{8 \pi^{2}}, \\
& F_{3}(0, \hat{\mu}) \approx \ln \frac{y}{\pi^{2}}+2 \gamma_{E}+\mathcal{D}\left(\frac{\hat{\mu}}{\pi}\right)=\ln \frac{y}{16 \pi^{2}}-\left[\psi\left(\frac{1}{2}+i \frac{\hat{\mu}}{2 \pi}\right)+\psi\left(\frac{1}{2}-i \frac{\hat{\mu}}{2 \pi}\right)\right],
\end{aligned}
$$

where " $\approx$ " denotes that the logarithmic divergence displayed on the right-hand side needs to be subtracted before setting $y \rightarrow 0$, and the function $\mathcal{D}$, which has the property $\mathcal{D}(0)=0$, corresponds to the notation in Ref. [40]. The analytic expression in terms of $\psi(z)=\Gamma^{\prime}(z) / \Gamma(z)$ comes from Ref. 20]. For $\hat{\mu} \neq 0$, the function $F_{4}$ diverges logarithmically at small $y$, but as it is always multiplied by $y$, this behaviour has little interest in the present context. Inserting the values in Eqs. (B7)-(B) into our expressions for $\alpha_{\mathrm{E} 1}^{\overline{\mathrm{MS}}}, \alpha_{\mathrm{E} 2}^{\overline{\mathrm{MS}}}, \alpha_{\mathrm{E} 7}^{\overline{\mathrm{MS}}}$, Eqs. (3.14), (3.15), (3.20) of Ref. 20] are reproduced.

For $y \rightarrow \infty$ and $\hat{\mu}$ fixed, the functions $F_{1}, F_{2}, F_{3}$ vanish as $\exp (-\sqrt{y})$, the function $F_{4}$ as $\exp (-2 \sqrt{y})$, modulo a powerlike prefactor. An interesting limit is obtained, however, by setting $y, \hat{\mu}$ simultaneously to infinity but keeping the ratio $z \equiv y / \hat{\mu}^{2}=m^{2} / \mu^{2}$ fixed. This corresponds to setting the temperature to zero but keeping $m, \mu$ finite. Then

$$
\begin{aligned}
\lim _{T \rightarrow 0} T^{4} F_{1}\left(\frac{m^{2}}{T^{2}}, \frac{\mu}{T}\right) & =\theta(1-z) \frac{\mu^{4}}{96 \pi^{2}}\left(2 w^{3}-3 z f_{2}\right), \\
\lim _{T \rightarrow 0} T^{2} F_{2}\left(\frac{m^{2}}{T^{2}}, \frac{\mu}{T}\right) & =\theta(1-z) \frac{\mu^{2}}{8 \pi^{2}} f_{2}, \\
\lim _{T \rightarrow 0} F_{3}\left(\frac{m^{2}}{T^{2}}, \frac{\mu}{T}\right) & =\theta(1-z) \frac{2}{z}\left(f_{2}-w\right), \\
\lim _{T \rightarrow 0} T^{2} F_{4}\left(\frac{m^{2}}{T^{2}}, \frac{\mu}{T}\right) & =\theta(1-z) \frac{\mu^{2}}{64 \pi^{4} z}\left(w^{4}-f_{2}^{2}\right),
\end{aligned}
$$

where

$$
w \equiv \sqrt{1-z}, \quad f_{2} \equiv \sqrt{1-z}-z \ln \frac{1+\sqrt{1-z}}{\sqrt{z}} .
$$

Inserting into our expressions for $\alpha_{\mathrm{E} 1}^{\overline{\mathrm{MS}}}, \alpha_{\mathrm{E} 2}^{\overline{\mathrm{MS}}}$, Eqs. (1) and (4) of Ref. [26] are reproduced.

Unfortunately the limit $\hat{\mu} \rightarrow 0$, of most interest to us in this paper, does not render any of the functions analytically

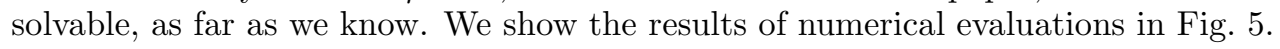

[1] M. Srednicki, R. Watkins and K.A. Olive, Nucl. Phys. B 310 (1988) 693.

[2] M. Hindmarsh and O. Philipsen, Phys. Rev. D 71 (2005) 087302 hep-ph/0501232.

[3] S. Dodelson and L.M. Widrow, Phys. Rev. Lett. 72 (1994) 17 hep-ph/9303287.

[4] K.N. Abazajian and G.M. Fuller, Phys. Rev. D 66 (2002) 023526 astro-ph/0204293; T. Asaka, S. Blanchet and M. Shaposhnikov, Phys. Lett. B 631 (2005) 151 hep-ph/0503065.

[5] D.J. Schwarz, Mod. Phys. Lett. A 13 (1998) 2771 gr-qc/9709027; N. Seto and J. Yokoyama, J. Phys. Soc. Jap. 72 (2003) 3082 gr-qc/0305096; L.A. Boyle and P.J. Steinhardt, astro-ph/0512014 

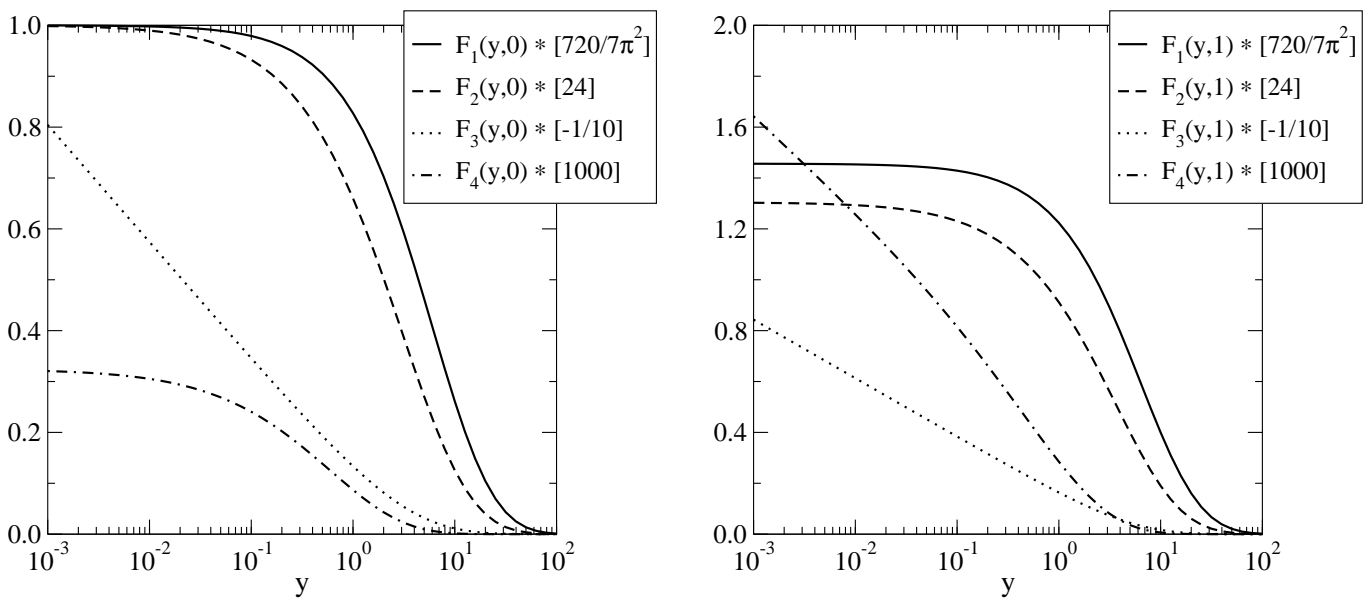

FIG. 5: The functions defined in Eqs. (B1) (B4), for $\hat{\mu}=0.0$ (left) and $\hat{\mu}=1.0$ (right) (all functions are even in $\hat{\mu}$ ). Note that the ranges of the vertical axes are different in the two plots.

[6] H. Davoudiasl, R. Kitano, G.D. Kribs, H. Murayama and P.J. Steinhardt, Phys. Rev. Lett. 93 (2004) 201301 hep-ph/0403019; W. Buchmüller, K. Hamaguchi, O. Lebedev and M. Ratz, Nucl. Phys. B 699 (2004) 292 hep-th/0404168; P. Brax, C. van de Bruck, A.C. Davis, J. Khoury and A. Weltman, Phys. Rev. D 70 (2004) 123518 astro-ph/0408415; G.L. Alberghi, R. Casadio and A. Tronconi, hep-ph/0310052

[7] U.W. Heinz, AIP Conf. Proc. 739 (2005) 163 nucl-th/0407067; P. Huovinen, Nucl. Phys. A 761 (2005) 296 nucl-th/0505036; R.S. Bhalerao, J.P. Blaizot, N. Borghini and J.Y. Ollitrault, Phys. Lett. B 627 (2005) 49 nucl-th/0508009.

[8] E.V. Shuryak, Sov. Phys. JETP 47 (1978) 212; S.A. Chin, Phys. Lett. B 78 (1978) 552.

[9] J.I. Kapusta, Nucl. Phys. B 148 (1979) 461.

[10] T. Toimela, Phys. Lett. B 124 (1983) 407.

[11] P. Arnold and C. Zhai, Phys. Rev. D 50 (1994) 7603 hep-ph/9408276; ibid. 51 (1995) 1906 hep-ph/9410360.

[12] C. Zhai and B. Kastening, Phys. Rev. D 52 (1995) 7232 hep-ph/9507380.

[13] K. Kajantie, M. Laine, K. Rummukainen and Y. Schröder, Phys. Rev. D 67 (2003) 105008 hep-ph/0211321].

[14] A.D. Linde, Phys. Lett. B 96 (1980) 289.

[15] D.J. Gross, R.D. Pisarski and L.G. Yaffe, Rev. Mod. Phys. 53 (1981) 43.

[16] A. Hietanen, K. Kajantie, M. Laine, K. Rummukainen and Y. Schröder, JHEP 01 (2005) 013 hep-lat/0412008.

[17] F. Di Renzo, M. Laine, V. Miccio, Y. Schröder and C. Torrero, hep-ph/0605042

[18] G.D. Moore, JHEP 10 (2002) 055 hep-ph/0209190; A. Ipp, G.D. Moore and A. Rebhan, JHEP 01 (2003) 037 hep-ph/0301057.

[19] A. Vuorinen, Phys. Rev. D 67 (2003) 074032 hep-ph/0212283.

[20] A. Vuorinen, Phys. Rev. D 68 (2003) 054017 hep-ph/0305183.

[21] A. Ipp and A. Rebhan, JHEP 06 (2003) 032 hep-ph/0305030; A. Ipp, A. Rebhan and A. Vuorinen, Phys. Rev. D 69 (2004) 077901 hep-ph/0311200.

[22] A. Gynther and M. Vepsäläinen, JHEP 01 (2006) 060 hep-ph/0510375; JHEP 03 (2006) 011 hep-ph/0512177.

[23] J.P. Blaizot, E. Iancu and A. Rebhan, Phys. Rev. D 68 (2003) 025011 hep-ph/0303045.

[24] G. Cvetič and R. Kögerler, Phys. Rev. D 70 (2004) 114016 hep-ph/0406028; M. Inui, A. Niegawa and H. Ozaki, Prog. Theor. Phys. 115 (2006) 411 hep-ph/0501277.

[25] X. Wang and J. Li, Commun. Theor. Phys. 33 (2000) 253 hep-ph/9804246.

[26] E.S. Fraga and P. Romatschke, Phys. Rev. D 71 (2005) 105014 hep-ph/0412298.

[27] M. Laine and M. Vepsäläinen, JHEP 02 (2004) 004 hep-ph/0311268.

[28] J. Ignatius, K. Kajantie, H. Kurki-Suonio and M. Laine, Phys. Rev. D 50 (1994) 3738 hep-ph/9405336.

[29] P. Ginsparg, Nucl. Phys. B 170 (1980) 388; T. Appelquist and R.D. Pisarski, Phys. Rev. D 23 (1981) 2305.

[30] K. Kajantie, M. Laine, K. Rummukainen and M. Shaposhnikov, Nucl. Phys. B 458 (1996) 90 hep-ph/9508379.

[31] E. Braaten and A. Nieto, Phys. Rev. D 53 (1996) 3421 hep-ph/9510408.

[32] K. Kajantie, M. Laine, K. Rummukainen and Y. Schröder, JHEP 04 (2003) 036 hep-ph/0304048.

[33] K. Rummukainen, private communication.

[34] S. Eidelman et al. [Particle Data Group], Phys. Lett. B 592 (2004) 1.

[35] K. Kajantie, M. Laine, K. Rummukainen and M. Shaposhnikov, Nucl. Phys. B 503 (1997) 357 hep-ph/9704416.

[36] M. Laine and O. Philipsen, Phys. Lett. B 459 (1999) 259 hep-lat/9905004; A. Hart and O. Philipsen, Nucl. Phys. B 572 (2000) 243 hep-lat/9908041.

[37] M. Laine and Y. Schröder, JHEP 03 (2005) 067 hep-ph/0503061.

[38] G. Boyd, J. Engels, F. Karsch, E. Laermann, C. Legeland, M. Lütgemeier and B. Petersson, Nucl. Phys. B 469 (1996) 419 
hep-lat/9602007.

[39] B. Beinlich, F. Karsch, E. Laermann and A. Peikert, Eur. Phys. J. C 6 (1999) 133 hep-lat/9707023; M. Okamoto et al. [CP-PACS Collaboration], Phys. Rev. D 60 (1999) 094510 hep-lat/9905005; Y. Namekawa et al. [CP-PACS Collaboration], Phys. Rev. D 64 (2001) 074507 hep-lat/0105012 ; R.V. Gavai, S. Gupta and S. Mukherjee, Phys. Rev. D 71 (2005) 074013 hep-lat/0412036.

[40] A. Hart, M. Laine and O. Philipsen, Nucl. Phys. B 586 (2000) 443 hep-ph/0004060.

[41] P. Giovannangeli and C.P. Korthals Altes, Nucl. Phys. B 721 (2005) 1 hep-ph/0212298; ibid. 721 (2005) 25 hep-ph/0412322.

[42] P. Bialas, A. Morel, B. Petersson, K. Petrov and T. Reisz, Nucl. Phys. B 581 (2000) 477 hep-lat/0003004; ibid. 603 (2001) 369 hep-lat/0012019.

[43] F. Karsch, K. Redlich and A. Tawfik, Eur. Phys. J. C 29 (2003) 549 hep-ph/0303108.

[44] A. Ali Khan et al. [CP-PACS collaboration], Phys. Rev. D 64 (2001) 074510 hep-lat/0103028.

[45] C. Bernard et al., PoS LAT2005 (2005) 156 hep-lat/0509053.

[46] Y. Aoki, Z. Fodor, S.D. Katz and K.K. Szabo, JHEP 01 (2006) 089 hep-lat/0510084.

[47] S. Ejiri, F. Karsch, E. Laermann and C. Schmidt, Phys. Rev. D 73 (2006) 054506 hep-lat/0512040.

[48] K. Kajantie, M. Laine, K. Rummukainen and M.E. Shaposhnikov, Phys. Rev. Lett. 77 (1996) 2887 hep-ph/9605288.

[49] K. Kajantie, M. Laine, K. Rummukainen and M.E. Shaposhnikov, Nucl. Phys. B 493 (1997) 413 hep-lat/9612006.

[50] P. Arnold and O. Espinosa, Phys. Rev. D 47 (1993) 3546 hep-ph/9212235; ibid. 50 (1994) 6662 (Erratum); Z. Fodor and A. Hebecker, Nucl. Phys. B 432 (1994) 127 hep-ph/9403219.

[51] G.D. Moore and D. Teaney, Phys. Rev. C 71 (2005) 064904 hep-ph/0412346; P. Petreczky and D. Teaney, Phys. Rev. D 73 (2006) 014508 hep-ph/0507318; H. van Hees, V. Greco and R. Rapp, hep-ph/0601166

[52] R.R. Parwani, Phys. Rev. D 45 (1992) 4695 hep-ph/9204216]; ibid. 48 (1993) 5965 (Erratum); A. Jakovác, Phys. Rev. D 53 (1996) 4538 hep-ph/9502313; A.I. Bugrii and V.N. Shadura, hep-th/9510232

[53] M. Laine and M. Losada, Nucl. Phys. B 582 (2000) 277 hep-ph/0003111. 